\title{
A New Expression for the Gain-Noise Relation of Single Carrier Avalanche Photodiodes with Arbitrary Staircase Multiplication Regions
}

\author{
A. Pilotto, P. Palestri, L. Selmi, M. Antonelli, F. Arfelli, G. Biasiol, G. Cautero, \\ F. Driussi, R. H. Menk, C. Nichetti, T. Steinhartova
}

\begin{abstract}
We propose a simple expression to relate the total excess noise factor of a single carrier multiplication staircase avalanche photodiode to the excess noise factor and gain given by the individual conduction band discontinuities. The formula is valid when electron impact ionization dominates hole impact ionization; hence, it is especially suited for staircase APDs with In-rich multiplication regions, as opposed, for example, to GaAs/AlGaAs systems where hole ionization plays an important role. The formula has been verified by accurate means of numerical simulations based on a newly developed nonlocal history dependent impact ionization model.
\end{abstract}

Index Terms-Avalanche Photodiodes, Impact Ionization, Excess Noise Factor

\section{INTRODUCTION}

The internal impact ionization gain $(M)$ in avalanche photodiodes (APDs) has an associated multiplication noise expressed by the excess noise factor $(F)$, which is the ratio between the power spectrum of the current noise and the

Manuscript received December 20, 2018; revised February 15, 2019; accepted February 18, 2019. Date of publication March 8, 2019; date of current version March 22, 2019. This work was supported by the Italian MIUR through the PRIN 2015 Project under Grant 2015WMZ5C8. The review of this paper was arranged by Editor G. Ghione. (Corresponding author: A. Pilotto.)

A. Pilotto and P. Palestri are with the Dipartimento Politecnico di Ingegneria e Architettura, University of Udine, 33100 Udine, Italy (e-mail: pilotto.alessandro@spes.uniud.it).

L. Selmi iswith the Dipartimento di Ingegneria Enzo Ferrari, University of Modena and Reggio Emilia, 41100 Modena, Italy.

M. Antonelli is with the Elettra-Sincrotrone Trieste S.C.p.A., 34149 Trieste, Italy.

F. Arfelli is with the Department of Physics, University of Trieste, 34128 Trieste, Italy, and also with the Istituto Nazionale di Fisica Nucleare, INFN Sezione di Trieste, 34100 Trieste, Italy.

G. Biasiol is with IOM CNR, Laboratorio TASC, 34149 Trieste, Italy.

G. Cautero is with the Elettra-Sincrotrone Trieste S.C.p.A., 34149 Trieste, Italy, and also with the Istituto Nazionale di Fisica Nucleare, INFN Sezione di Trieste, 34100 Trieste, Italy.

F. Driussi is with the Dipartimento Politecnico di Ingegneria e Architettura, University of Udine, 33100 Udine, Italy.

R. H. Menk is with the Elettra-Sincrotrone Trieste S.C.p.A., 34149 Trieste, Italy, also with the Istituto Nazionale di Fisica Nucleare, INFN Sezione di Trieste, 34100 Trieste, Italy, and also with the Department of Medical Imaging, University of Saskatchewan, Saskatoon, SK S7N 5A2, Canada.

C. Nichetti is with the Elettra-Sincrotrone Trieste S.C.p.A., 34149 Trieste, Italy, and also with the Department of Physics, University of Trieste, 34128 Trieste, Italy.

T. Steinhartova is with the Department of Physics, University of Trieste, 34128 Trieste, Italy, and also with IOM CNR, Laboratorio TASC, 34149 Trieste, Italy.

Color versions of one or more of the figures in this paper are available online at http://ieeexplore.ieee.org.

Digital Object Identifier 10.1109/TED.2019.2900743 power spectrum of pure shot noise. To achieve a low $F$, the semiconductor material in the multiplication region should have very different electron and hole impact ionization (II) coefficients, $\alpha$ and $\beta$ respectively [1]. This is not the case for most III-V compound semiconductors. However, by tailoring the conduction and valence band profiles of heterojunction superlattice structures (called staircase APDs), it is possible to create large conduction band steps with modest perturbation of the valence band continuity, thus enhancing electron II with respect to hole II [2]. In addition, for near infrared optical fiber communication links, the detector performance can be improved by using InAs alloys, where $\alpha$ is inherently much larger than $\beta$ [3]. A notable example of such a device is the Separate Absorption and Multiplication (SAM) single step staircase APD proposed in [4].

The accurate prediction of gain and excess noise factor is fundamental for device optimization. To reach this objective, many different approaches have been proposed in the literature, ranging from the Monte Carlo solution of the Boltzmann Transport equation with a detailed description of electronphonon and impact ionization scattering mechanisms [5], [6], to numerical local or nonlocal models that relate $M$ and $F$ to the spatial profiles of $\alpha$ and $\beta$ [7], [8], [9], to simplified analytical formulas that can guide the design of APDs [2], [10].

For a staircase structure with $N$ identical steps, and assuming that multiplication is dominated by electron II (so that hole II can be neglected), a simple analytical formula for the total excess noise factor has been derived in [2]:

$$
F_{T O T}=1+\frac{\delta\left[1-(2-\delta)^{-N}\right]}{(2-\delta)}
$$

where $\delta$ is the fraction of electrons that do not ionize at each step and thus $2-\delta$ is the gain per step. The total gain, defined as the number of charges flowing out of the staircase normalized to the number of those entering it, is then $M_{T O T}=(2-\delta)^{N}$. A model accounting for hole II due to the electric field between the steps has been proposed in [10] based on the theory in [11] and assuming that all steps have the same amplitude. A large electric field between the steps is needed in $\mathrm{AlGaAs} / \mathrm{GaAs}$ systems where the conduction band discontinuity is in the order of $0.5 \mathrm{eV}$ and the energy gap is larger than $1 \mathrm{eV}$ [9]. On the other hand, in InAsSb structures [4], steps as large as $0.6 \mathrm{eV}$ can be fabricated, that are more than twice the corresponding energy gap $(\approx 0.25 \mathrm{eV})$. 
(c) 2019 IEEE. Personal use of this material is permitted. Permission from IEEE must be obtained for all other users, including reprinting/ republishing this material for advertising or promotional purposes, creating new collective works for resale or redistribution to servers or lists, or reuse of any copyrighted components of this work in other works. doi: 10.1109/TED.2019.2900743 - (C)2019 IEEE. Personal use is permitted, but republication/redistribution requires IEEE permission.

See http://www.ieee.org/publications_standards/publications/rights/index.html for more information.

In this paper, we extend previous analyses by deriving a generalization of Eq. 1 (namely: Eq. 5) valid for single carrier multiplication staircase APDs with arbitrary step gains $\left(M_{i}\right)$ and step multiplication noise $\left(F_{i}\right)$. The expression is suited to compute the overall $M_{T O T}$ and $F_{T O T}$ and to optimize arbitrary ladders of non uniform layers. Using this generalized formula and accurate numerical models, it is found that for given gain, structures with non uniform energy steps can reduce the overall excess noise factor $F_{T O T}$ if the steps with a large amplitude of the conduction band discontinuity lie ahead along the direction of carrier flow.

The paper proceeds as follows. The new formula is derived in Section II. The nonlocal history dependent model used to verify the formula is described in Section III together with its calibration. Results from the numerical model and the analytical formula are reported in Section IV. Conclusions are finally drawn in Section V.

\section{DERIVATION OF THE EQUATION FOR THE EXCESS NOISE FACTOR}

Let's consider, for the sake of simplicity, a two-steps staircase APD with total gain $M_{T O T}$. By definition the total excess noise factor is given by $F_{T O T}=\left\langle m_{T O T}^{2}\right\rangle /\left\langle m_{T O T}\right\rangle^{2}$, where $m_{T O T}$ is the random process that represents the multiplication and $\left\langle m_{T O T}\right\rangle=M_{T O T}$ is the ensemble average of $m_{T O T}$. If we denote as $M_{1}$ and $M_{2}$ the gain of the first and the second step respectively, such that $M_{T O T}=M_{1} M_{2}$, then the computation of $\left\langle m_{T O T}^{2}\right\rangle$ yields:

$$
\left\langle m_{T O T}^{2}\right\rangle=\left\langle m_{2}^{2}\right\rangle M_{1}+\left\langle m_{1}^{2}\right\rangle M_{2}^{2}-M_{1} M_{2}^{2}
$$

Interestingly, Eq. 2 can be rewritten as:

$$
\begin{aligned}
\left\langle\left(m_{\text {TOT }}-M_{\text {TOT }}\right)^{2}\right\rangle= & \left\langle\left(m_{2}-M_{2}\right)^{2}\right\rangle M_{1} \\
& +\left\langle\left(m_{1}-M_{1}\right)^{2}\right\rangle M_{2}^{2}
\end{aligned}
$$

which is consistent with Eq. 2.3 in [12] and with Eq. 38 in [10] for photomultiplier tubes. Eq. 2 allows then to write the total excess noise factor as:

$$
F_{T O T}=F_{1}+\frac{F_{2}-1}{M_{1}}
$$

The extension of Eq. 4 to the case of a N-steps staircase structure (see Fig. 1) is straightforward:

$$
F_{T O T}=F_{1}+\sum_{i=2}^{N} \frac{F_{i}-1}{\prod_{k=1}^{i-1} M_{k}}
$$

It is easy to show that Eq. 5 gives the same result of Eq. 1 when all the steps have gain $M_{i}=(2-\delta)$ and excess noise factor $F_{i}=1+\delta\left[1-(2-\delta)^{-1}\right] /(2-\delta)$ as per Eq. 1. However, differently from Eq. 1, Eq. 5 is valid also in structures with an arbitrary sequence of decreasing steps and thus useful to optimize arbitrary staircase APDs.

Similarly to the well known Friis formula for the noise figure of cascaded amplifiers [13], Eq. 5 tells us that the gain of the first step has a critical role in achieving low noise. The first step must have high gain and low excess noise, two conditions that can be achieved only with large band offsets, as it will be seen in Section IV.

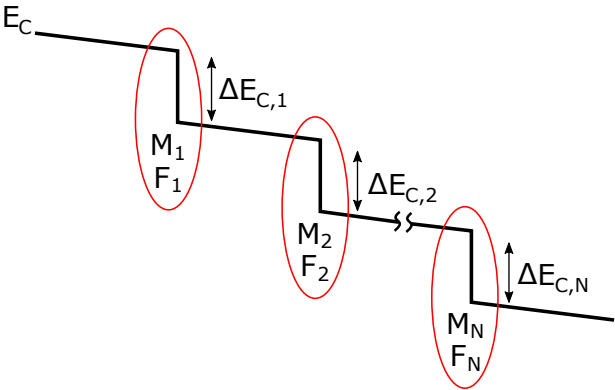

Fig. 1. Conduction band profile of a staircase structure with $\mathrm{N}$ steps. $M_{i}$ and $F_{i}$ are the gain and excess noise factor of each step.

It is worth noting that Eq. 5 stays valid also in the more general case where $M_{1}$ and $F_{1}$ represent the e-h pair generation due to an incoming high-energy photon (e.g. a X-ray) provided that we interpret $M_{1}$ as the average number of e-h pairs per photon and $F_{1}=1+f / M_{1}$ where $f$ is the so called Fano factor ${ }^{1}$ [14]. Under these circumstances, Eq. 5 yields to the same formula proposed in [14] for the excess noise of APDs used for X-ray detection.

Compared to existing numerical expressions, Eq.5 can be used as an effective tool to combine simulations of single steps and eventually find the optimum sequence of steps for maximum gain and low noise, as we will see in Section IV.

\section{Model And CALibration}

Eq. 5 must be coupled to an impact ionization model for each step in the ladder suited to compute the gains $M_{i}$ accurately. To this end we use the nonlocal history dependent model of [9]. This model follows the framework proposed in [8] to relate the ionization probability to gain and multiplication noise. The main distinctive factor with respect to [8] lies in the definition of the effective field. By using a simple energy balance model, we define an effective field for electron impact ionization as:

$$
E_{e f f, e}\left(x \mid x^{\prime}\right)=\frac{1}{\lambda_{e}} \int_{x}^{x^{\prime}} \frac{d E_{C}}{d x}\left(x^{\prime \prime}\right) \exp \left(\frac{x^{\prime \prime}-x^{\prime}}{\lambda_{e}}\right) d x^{\prime \prime}
$$

where $E_{C}$ is the conduction band profile and $\lambda_{e}$ a suitable mean free path. The electron is generated at the position $x$ (optically or by II) and ionizes in $x^{\prime}$. The electron II probability per unit-length is then related to $E_{e f f, e}\left(x \mid x^{\prime}\right)$ by:

$$
\alpha\left(E_{\text {eff }, e}\right)\left(x \mid x^{\prime}\right)=A_{e} \cdot \exp \left(-\left(\frac{E_{c e}}{E_{e f f, e}\left(x \mid x^{\prime}\right)}\right)^{\gamma_{e}}\right)
$$

where $A_{e}, E_{c e}$ and $\gamma_{e}$ are the adjustable model parameters to be calibrated against the experimental data. Being based on an energy balance equation, the model will be referred in the following as Energy Balance History Dependent Model (EBHDM).

Our target is to calibrate the EBHDM on the experiments in [4] for a single step AlInAsSb/InAsSb APD where multiplication takes place in the $\operatorname{InAs}_{0.91} \mathrm{Sb}_{0.09}$ layer. To this end we start with InAs impact ionization parameters and then

\footnotetext{
${ }^{1}$ The Fano factor is the ratio between the variance of the generation process and the number of generated electron-hole pairs.
} 
adjust them for the small $\mathrm{Sb}$ fraction of the multiplication layer. In particular, the $\lambda_{e}$ parameter was extracted from the average electron velocity versus electric field characteristics reported in [15] for InAs. In fact, the energy balance equation at the basis of Eq.6 relates the average electron energy $W$, to the electric field $E$ in uniform structures by considering that the deviation of $W$ from the equilibirum energy $1.5 k_{B} T$ is proportional to $E$ via a suitable mean-free-path; in other words $W-1.5 k_{B} T=q \lambda E, q$ being the electron charge, $k_{B}$ the Boltzmann constant and $T$ the temperature. By inspecting the energy-field relation between $10^{4} \mathrm{~V} / \mathrm{cm}$ and $10^{6} \mathrm{~V} / \mathrm{cm}$ in [15], one can extract a mean value of $\lambda_{e}=73.7 \mathrm{~nm}$. The $A_{e}$, $E_{c e}$ and $\gamma_{e}$ parameters of Eq.7 are then adjusted to reproduce the gain and excess noise measured in [3] for InAs p-i-n diodes. A good match between experiments and simulations (Fig.2) is finally obtained by using $A_{e}=4.62 \times 10^{4} \mathrm{~cm}^{-1}$, $E_{c e}=1.39 \times 10^{5} \mathrm{~V} / \mathrm{cm}$ and $\gamma_{\rho}=0.378$ 「31.

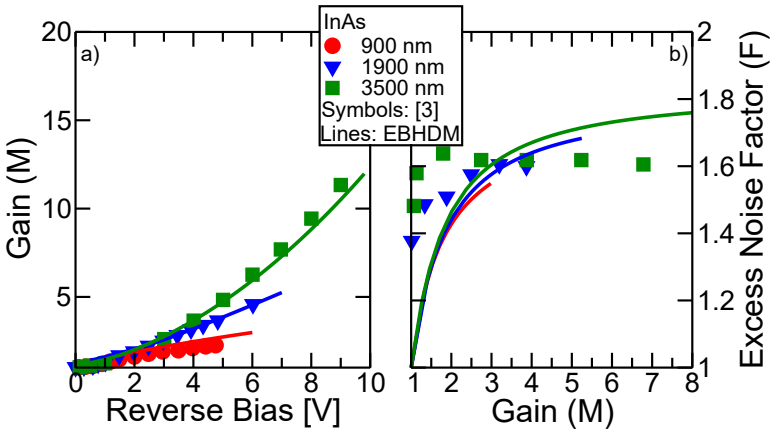

Fig. 2. Gain versus applied voltage (a) and excess noise factor versus gain (b) for InAs p-i-n diodes with different intrinsic region thicknesses $(900,1900$ and $3500 \mathrm{~nm}$ ). Experimental results from [3] (symbols) are compared with the EBHDM (lines).

Having calibrated the model on InAs, we can move to the device of [4], that is a SAM APD made of a 500$\mathrm{nm}$ i- $\mathrm{Al}_{0.7} \mathrm{In}_{0.3} \mathrm{As}_{0.31} \mathrm{Sb}_{0.69}$ absorption region followed by a single step multiplication region. The step of the staircase is composed by a $37-\mathrm{nm}$ graded $\mathrm{i}-\mathrm{Al}_{x} \mathrm{In}_{1-x} \mathrm{As}_{y} \mathrm{Sb}_{1-y}$, a 6$\mathrm{nm} \mathrm{i-InAs} \mathrm{As}_{0.91} \mathrm{Sb}_{0.09}$ and a 61-nm graded i- $\mathrm{Al}_{x} \mathrm{In}_{1-x} \mathrm{As}_{y} \mathrm{Sb}_{1-y}$ layers. The diode structure and the band-diagram are reported in Fig.3.

Since multiplication takes place in the $\operatorname{InAs} \mathrm{As}_{0.91} \mathrm{Sb}_{0.09}$ film, whose energy gap is $0.25 \mathrm{eV}$ in contrast to the $0.35 \mathrm{eV}$ of InAs [15], the parameter $E_{c e}$ was scaled accordingly by a factor of $0.35 / 0.25=1.4$, following the simple picture that the II rate depends exponentially on the energy gap of the material. The parameter $A_{e}$, instead, has been adjusted to match the measured gain and excess noise at low applied voltage in [4], see Fig.4. Compared to InAs, $A_{e}$ has been increased by a factor of 6.5 .

The deviation from experiments visible in Fig.4 for reverse bias higher than $3.0 \mathrm{~V}$ is in fact due to considering only electron II in the $\operatorname{InAs}_{0.91} \mathrm{Sb}_{0.09}$ multiplication layer.

Following this first calibration, the EBHDM can be applied to simulate multiplication at heterojunctions where impact ionization takes place in $\mathrm{InAs}_{0.91} \mathrm{Sb}_{0.09}$, as the one of Fig.3. In the next Section we will focus on the effect of the steps alone, with very low electric fields between the steps.
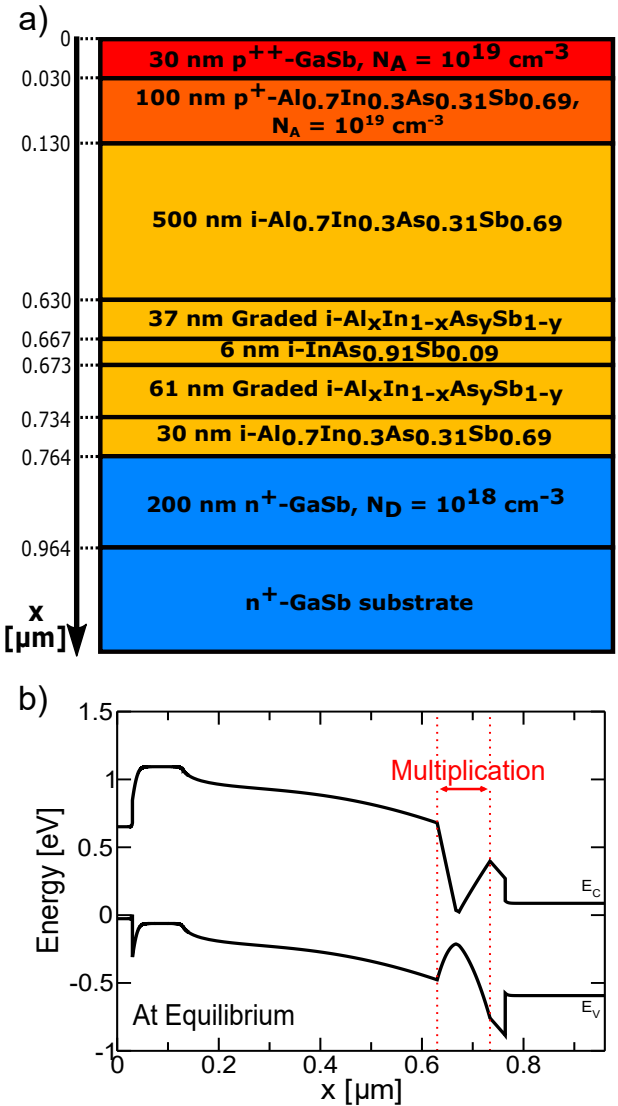

Fig. 3. (a) Sketch and (b) band diagram at equilibrium extracted from TCAD simulations [16] of the staircase SAM-APD reported in [4], along the vertical direction.

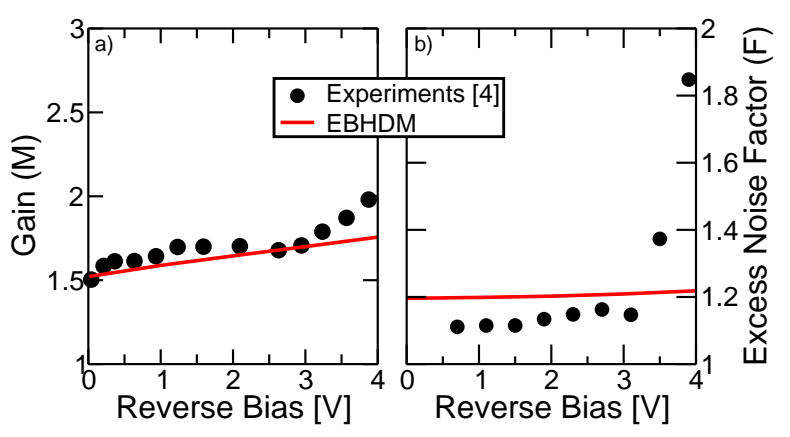

Fig. 4. Gain versus applied voltage (a) and excess noise factor versus applied voltage (b) for the staircase SAM-APD reported in [4]. Experimental results from [4] (symbols) are compared with the EBHDM (lines).

\section{NUMERICAL RESUlts}

We now use the EBHDM calibrated on $\operatorname{InAs}_{0.91} \mathrm{Sb}_{0.09}$ to verify the validity of Eq.5. We begin our analysis by simulating single steps with conduction band discontinuities of different amplitude $\left(\Delta E_{C}\right)$. In all simulations, we assume that the applied bias is the one that induces the electric field that perfectly compensates the quasi-field due to material grading between the steps, so that the conduction band energy is piecewise constant. As it can be seen in Fig.5, owing to the small gap of the multiplication region, $\Delta E_{C}$ values below 1 $\mathrm{eV}$ provide gains that tend to 2 . We also see that the relation 
(c) 2019 IEEE. Personal use of this material is permitted. Permission from IEEE must be obtained for all other users, including reprinting/ republishing this material for advertising or promotional purposes, creating new collective works for resale or redistribution to servers or lists, or reuse of any copyrighted components of this work in other works. doi: 10.1109/TED.2019.2900743 - (C)2019 IEEE. Personal use is permitted, but republication/redistribution requires IEEE permission.

See http://www.ieee.org/publications_standards/publications/rights/index.html for more information.

between the simulated gain and excess noise factor closely matches Eq.1 (for $N=1$ since we consider only one step here). Note that a local II model with electron ionization only would provide $F=2-1 / M$, that tends to 2 for large gains instead of the bell shape shown in Fig. 5 using the EBHDM. When we have steps with an electric field in between, the $F(M)$ curve obtained using the EBHDM [9], lies between the results obtained using Eq. 1 and the ones obtained using the Local Model in Fig. 5. This indicates that, regardless of the conduction band profile (strongly nonlocal as in a straircase or as uniform as possible as assumed by local models), if hole II is negligible, the room for optimization is limited in the $F=1$ to $F=2$ range. In Fig. 5, the same experimental data from [4], is reported too. We observe that measured values lie very close to the predictions of Eq. 1 except for high biases when hole impact ionization comes into play.

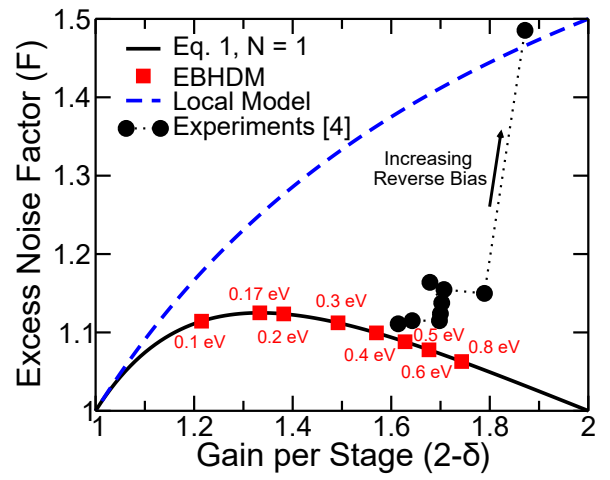

Fig. 5. Comparison between the excess noise factor versus gain curves predicted by Eq. 1 for $N=1$ (solid line), by the EBHDM (squares) and by the Local Model (blue dashed line) for single step staircase structures with different amplitude of the conduction band discontinuity. Experimental data from [4] is also reported (circles).

We have then considered staircase structures with different sequences of steps and compared the EBHDM with Eq.5. The results, reported in Tab.I, demonstrate a very good agreement with Eq.5, with errors well below $1 \%$.

We also see that for a given gain, it is advantageous to locate first the steps with the largest $\Delta E_{C}$. In fact, consistently with Eq.5, the noise added by each step is divided by the gain of the preceding steps. Since the order of the steps has no effect on the gain, the sequence can be optimized to minimize $F$. However, due to the small range of $F$ values covered by individual abrupt steps (Fig.5), the reduction of $F$ offered by optimized ordering of the steps is limited, unless the number of steps and the difference among them is large (last two rows of Tab.I).

To visualize how the excess noise and gain build up along the structure, we plot $M(x)$ and $F(x)$ in Fig.6 as a function of the position where the initial carrier is generated. We consider the two cases with identical gain $M=57.23$ in the last two rows of Tab.I. As expected, the gain decreases moving inside the staircase, i. e. when electrons are generated inside the structures, since one loses the gain associated to the previous steps. As for the excess noise, the spatial distribution changes remarkably with the ordering of the steps. If the steps with larger $\Delta E_{C}$ come first, the excess noise increases while moving inside the multiplication region, since the high noise

\begin{tabular}{|c|c|c|c|c|c|}
\hline $\mathrm{N}$ & $\Delta E_{C}[\mathrm{eV}]$ & $\mathrm{M}(\mathrm{sim})$. & $\mathrm{F}(\mathrm{sim})$. & $\mathrm{F}$ (Eq.5) & \% error \\
\hline 5 & 0.6 (uniform) & 13.20 & 1.179 & 1.178 & 0.085 \\
\hline 2 & $0.17,0.6$ & 2.25 & 1.180 & 1.183 & 0.254 \\
\hline 2 & $0.6,0.17$ & 2.25 & 1.150 & 1.153 & 0.261 \\
\hline 5 & $0.2,0.3,0.4,0.5,0.6$ & 8.85 & 1.293 & 1.295 & 0.155 \\
\hline 5 & $0.6,0.5,0.4,0.3,0.2$ & 8.85 & 1.211 & 1.212 & 0.083 \\
\hline 5 & $0.2,0.2,0.2,0.2,0.6$ & 6.14 & 1.342 & 1.344 & 0.149 \\
\hline 5 & $0.2,0.2,0.6,0.2,0.2$ & 6.14 & 1.317 & 1.319 & 0.152 \\
\hline 5 & $0.6,0.2,0.2,0.2,0.2$ & 6.14 & 1.268 & 1.270 & 0.157 \\
\hline 10 & $5 \times 0.17$ and $5 \times 0.6$ & 57.23 & 1.415 & 1.420 & 0.353 \\
\hline 10 & $5 \times 0.6$ and $5 \times 0.17$ & 57.23 & 1.207 & 1.209 & 0.166 \\
\hline
\end{tabular}

TABLE I

COMPARISON BETWEEN THE EXCESS NOISE FACTOR OBTAINED WITH THE EBHDM AND THE ONE CALCULATED USING EQ.5 FOR STAIRCASE STRUCTURES WITH DIFFERENT NUMBER OF STEPS AND CONDUCTION BAND DISCONTINUITIES WITH DIFFERENT AMPLITUDES.

of the last stages gets divided by a gain which gets lower and lower; thus we do not take advantage of the high gain provided by the initial steps.

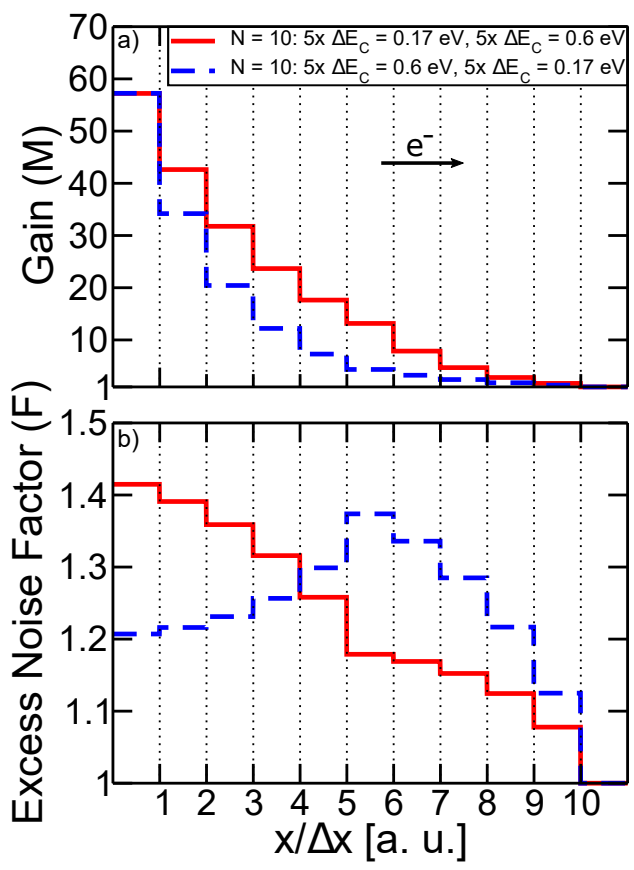

Fig. 6. Gain (a) and excess noise factor (b) as a function of the normalized position $x / \Delta x$ ( $\Delta x$ being the step's length) at which an electron is generated for two staircase structures with ten steps. Solid red line: five steps with $\Delta E_{C}=0.17 \mathrm{eV}$ are followed by five steps with $\Delta E_{C}=0.6 \mathrm{eV}$. Dashed blue line: five steps with $\Delta E_{C}=0.6 \mathrm{eV}$ are followed by five steps with $\Delta E_{C}=0.17 \mathrm{eV}$.

To assess the influence of the sequence of steps on the device bandwidth, we run calculations using the Random Path Length algorithm presented in [17], which is an improved version, consistent with the definition of effective field of Eq. 6, of the one initially proposed by [18], [19]. We considered the devices with 4 steps of $0.2 \mathrm{eV}$ and one step of $0.6 \mathrm{eV}$ (7th, 8th and 9th rows in Tab. I) all having the same gain of 6.14. The highest bandwidth is obtained when the large step lies in the middle of the sequence. If the large step is at the beginning the bandwidth is $5 \%$ lower, if it is at the end, the bandwidth is $10 \%$ lower. Consider that in all cases the total duration of the current pulse is the same and only depends on the number of steps and their spacing (see Fig. 7). The rising part of the current 
(c) 2019 IEEE. Personal use of this material is permitted. Permission from IEEE must be obtained for all other users, including reprinting/ republishing this material for advertising or promotional purposes, creating new collective works for resale or redistribution to servers or lists, or reuse of any copyrighted components of this work in other works. doi: 10.1109/TED.2019.2900743 - (C)2019 IEEE. Personal use is permitted, but republication/redistribution requires IEEE permission.

See http://www.ieee.org/publications_standards/publications/rights/index.html for more information.

waveform has different shapes depending on the position of the largest step. If the latter is at the beginning, the waveform is steep at the beginning and then slows down, the opposite if it is at the end; when it is in the middle the steepness is more uniform, with advantages in terms of bandwidth. Note that the falling portion of the waveform is faster in the cases where the largest step is at the beginning or in the middle, resulting in better bandwidth compared to the case when the largest step is at the end.

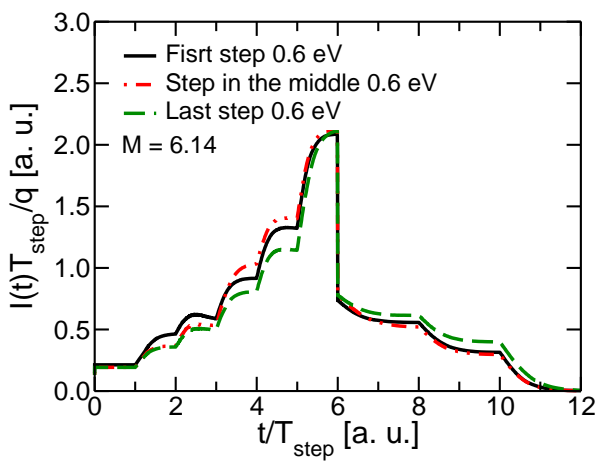

Fig. 7. Normalized current waveforms as a function of the normalized time $t / T_{\text {step }}$ for three different staircase structures with 5 steps: $1 \mathrm{x} \Delta E_{C}=0.6$ $\mathrm{eV}$ and $4 \mathrm{x} \Delta E_{C}=0.2 \mathrm{eV}$ (black solid line), $2 \mathrm{x} \Delta E_{C}=0.2 \mathrm{eV}, 1 \mathrm{x} \Delta E_{C}$ $=0.6 \mathrm{eV}$ and $2 \mathrm{x} \Delta E_{C}=0.2 \mathrm{eV}$ (red dashed line) and $4 \mathrm{x} \Delta E_{C}=0.2 \mathrm{eV}$ and $1 \mathrm{x} \Delta E_{C}=0.6 \mathrm{eV}$ (green dashed line). $T_{\text {step }}$ is the transit time across a single step of the staircase structure.

\section{Discussion AND Conclusion}

We have derived a simple formula for the excess noise in multi-step APDs with single carrier multiplication that extends the original model in [2] to the case of steps with different amplitude. The results allow technologists to optimize staircase APDs for best $F$ at given $M$, without need for more computationally demanding numerical models, such as Monte Carlo or history dependent models.

The formula suggests that, for minimum excess noise, larger conduction band steps should come before the small ones along the carrier path, this is consistent with the analysis for photomultiplier tubes reported in [10]. The formula for the excess noise has been validated by numerical simulations using a recently proposed nonlocal history dependent model for impact ionization. Although we considered here only cases where the effect of the electric field between the steps is negligible, the proposed formula could in principle be used also when this field is not null: essentially the region between two steps has its own gain and excess noise that can be combined with the gain and noise of the steps with Eq. 1 .

The main limitation of the proposed formula is that it is valid only when hole impact ionization is negligible. However, we would like to point out that the effects of hole II become relevant when the conduction band discontinuities do not provide electrons with sufficient energy to ionize, as for example in the $\mathrm{AlGaAs} / \mathrm{GaAs}$ system [9]. When this happens, multiplication in the regions between the steps tends to have the same impact as the one at the steps. In these conditions, the use of different sequences of steps with different amplitudes would likely have less effect on the overall gain and excess noise. Hence, one should either use the equation proposed in [10] to fit experimental data (as in [20]), or should resort to fully numerical models.

\section{ACKNOWLEDGMENTS}

This work was supported by Italian MIUR through the PRIN 2015 Project under Grant 2015WMZ5C8.

\section{REFERENCES}

[1] R. J. McIntyre, "Multiplication noise in uniform avalanche diodes", IEEE Transactions on Electron Devices, vol. ED-13, no. 1, pp. 164168, Jan. 1966. doi: 10.1109/T-ED.1966.15651.

[2] F. Capasso, W. T. Tsang, G. F. Williams, "Staircase solid-state photomultipliers and avalanche photodiodes with enhanched ionization rates ratio", IEEE Transactions on Electron Devices, vol. ED-30, no.4, pp. 381-390, Apr. 1983. doi: 10.1109/T-ED.1983.21132.

[3] A. R. J. Marshall, J. P. R. David, C. H. Tan, "Impact ionization in InAs electron avalanche photodiodes", IEEE Transactions on Electron Devices, vol. 57, no. 10, pp. 2631-2638, Oct. 2010. doi: 10.1109/TED.2010.2058330.

[4] M. Ren, S. Maddox, Y. Chen, M. Woodson, J. C. Campbell, S. Banks, "AlInAsSb/GaSb staircase avalanche photodiode", Applied Physics Letters, vol. 108, no. 8, pp. 081101-1-081101-4, Feb. 2016. doi: 10.1063/1.4942370.

[5] D. S. Ong, K. F. Li, S. A. Plimmer, G. J. Rees, J. P. R. David, P. N. Robson, "Full band Monte Carlo modeling of impact ionization, avalanche multiplication, and noise in submicron GaAs $p+-i-n+$ diodes”, Journal of Applied Physics, vol. 87, no. 11, pp. 7885-7891, Jun. 2000. doi: 10.1063/1.373472.

[6] F. Ma, S. Wang, X. Li, A. Anselm, X. G. Zheng, A. L. Holmes Jr., J. C. Campbell, "Monte Carlo simulation of low-noise avalanche photodiodes with heterojunctions”, Journal of Applied Physics, vol. 92, no. 8, pp 4791-4795, Oct. 2002. doi: 10.1063/1.1505987.

[7] M. M. Hayat, W. L. Sargeant, B. E. A. Saleh, "Effect of dead space on gain and noise in Si and GaAs avalanche photodiodes”, IEEE Journal of Quantum Electronics, vol. 28, no. 5, pp. 1360-1365, May 1992. doi: 10.1109/3.135278.

[8] R. J. McIntyre, “A new look at impact ionization - part I: a theory of gain, noise, breakdown, and frequency response”, IEEE Transactions on Electron Devices, vol. 46, no. 8, pp. 1623-1631, Aug. 1999. doi: 10.1109/16.777150.

[9] C. Nichetti, A. Pilotto, P. Palestri, L. Selmi, M. Antonelli, F. Arfelli, G. Biasiol, G. Cautero, F. Driussi, N. Y. Klein, R. H. Menk, T. Steinhartova, "An improved nonlocal history dependent model for gain and noise in avalanche photodiodes", IEEE Transactions on Electron Devices, vol. 65, no. 5, pp. 1823-1829, May 2018. doi: 10.1109/TED.2018.2817509.

[10] M. C. Teich, K. Matsuo, B. E. A. Saleh, "Excess noise factors for conventional and superlattice avalanche photodiodes and photomultiplier tubes”, IEEE Journal of Quantum Electronics, vol. QE-22, no. 8, pp. 1184-1193, Aug. 1986. doi: 10.1109/JQE.1986.1073137.

[11] K. M. Van Vliet, A. Friedman, L. M. Rucker, "Theory of carrier multiplication and noise in avalanche devices - part II: two-carrier processes", IEEE Transactions on Electron Devices, vol. ED-26, no. 5, pp. 752-764, May. 1979. doi: 10.1109/t-ed.1979.19490.

[12] R. E. Burgess, "Some topics in the fluctuations of photo-processes in solids", Journal of Physics and Chemistry of Solids, vol. 22, pp. 371377, 1961. doi: 10.1016/0022-3697(61)90284-0.

[13] B. Razavi, “RF Microelectronics, 2nd edition”, Prentice Hall, 2012.

[14] C. H. Tan, R. B. Gomes, J. P. R. David, A. M. Barnett, D. J. Bassford, J. E. Lees, J. S. Ng, “Avalanche gain and energy resolution of semiconductor X-ray detectors", IEEE Transactions on Electron Devices, vol. 58, no. 6, pp. 1696-1701, Jun. 2011. doi: 10.1109/TED.2011.2121915.

[15] M. V. Fischetti, "Monte Carlo transport in technologically significant semiconductors of the diamond and zinc-blende structures - part I: homogeneous transport", IEEE Transactions on Electron Devices, vol. 38, no. 3, pp. 634-649, Mar. 1991. doi: 10.1109/16.123508.

[16] Synopsys, "Sentaurus Device user guide”, version L-2016.03, 2016.

[17] A. Pilotto, P. Palestri, L. Selmi, M. Antonelli, F. Arfelli, G. Biasiol, G. Cautero, F. Driussi, R. H. Menk, C. Nichetti, T. Steinhartova, "An improved Random Path Length algorithm for $p-i-n$ and staircase avalanche photodiodes", Proceedings of SISPAD 2018, pp. 26-30, Sep. 2018. doi: 10.1109/SISPAD.2018.8551751. 
(c) 2019 IEEE. Personal use of this material is permitted. Permission from IEEE must be obtained for all other users, including reprinting/ republishing this material for advertising or promotional purposes, creating new collective works for resale or redistribution to servers or lists, or reuse of any copyrighted components of this work in other works.

doi: 10.1109/TED.2019.2900743 - (C)2019 IEEE. Personal use is permitted, but republication/redistribution requires IEEE permission.

See http://www.ieee.org/publications_standards/publications/rights/index.html for more information.

[18] D. S. Ong, K. F. Li, G. J. Rees, J. P. R. David, P. N. Robson, “A simple model to determine multiplication and noise in avalanche photodiodes", Journal of Applied Physics, vol. 83, no. 6, pp. 3426-3428, Mar. 1998. doi: $10.1063 / 1.367111$

[19] J. S. Ng, C. H. Tan, B. K. Ng, P. J. Hambleton, J. P. R. David, G. J. Rees, A. H. You, D. S. Ong, "Effect of dead space on avalanche speed", IEEE Transactions on Electron Devices, vol. 49, no. 4, pp. 544-549, Apr. 2002. doi: 10.1109/16.992860.

[20] G. M. Williams, M. Compton, D. A. Ramirez, M. M. Hayat, A. S. Huntington, "Multi-Gain-Stage InGaAs avalanche photodiode with enhanced gain and reduced excess noise", IEEE Journal of the Electron Device Society, vol. 1, no. 2, pp. 54-65, Feb. 2013. doi: 10.1109/JEDS.2013.2258072. 